\title{
A new proof of the sharpness of the phase transition for Bernoulli percolation on $\mathbb{Z}^{d}$
}

\author{
Hugo Duminil-Copin and Vincent Tassion
}

February 11, 2015

\begin{abstract}
We provide a new proof of the sharpness of the phase transition for nearest-neighbour Bernoulli percolation. More precisely, we show that

- for $p<p_{c}$, the probability that the origin is connected by an open path to distance $n$ decays exponentially fast in $n$.

- for $p>p_{c}$, the probability that the origin belongs to an infinite cluster satisfies the mean-field lower bound $\theta(p) \geq \frac{p-p_{c}}{p\left(1-p_{c}\right)}$.

This note presents the argument of [DCT15, which is valid for long-range Bernoulli percolation (and for the Ising model) on arbitrary transitive graphs in the simpler framework of nearest-neighbour Bernoulli percolation on $\mathbb{Z}^{d}$.
\end{abstract}

\section{Statement of the result}

Notation. Fix an integer $d \geq 2$. We consider the $d$-dimensional hypercubic lattice $\left(\mathbb{Z}^{d}, \mathbb{E}^{d}\right)$. Let $\Lambda_{n}=\{-n, \ldots, n\}^{d}$, and let $\partial \Lambda_{n}:=\Lambda_{n} \backslash \Lambda_{n-1}$ be its vertex-boundary. Throughout this note, $S$ always stands for a $\mathrm{fi}$ nite set of vertices containing the origin. Given such a set, we denote its edge-boundary by $\Delta S$, defined by all the edges $\{x, y\}$ with $x \in S$ and $y \notin S$.

Consider the Bernoulli bond percolation measure $\mathbb{P}_{p}$ on $\{0,1\} \mathbb{E}^{d}$ for which each edge of $\mathbb{E}^{d}$ is declared open with probability $p$ and closed otherwise, independently for different edges.

Two vertices $x$ and $y$ are connected in $S \subset V$ if there exists a path of vertices $\left(v_{k}\right)_{0 \leq k \leq K}$ in $S$ such that $v_{0}=x, v_{K}=y$, and $\left\{v_{k}, v_{k+1}\right\}$ is open for every $0 \leq k<K$. We denote this event by $x \stackrel{S}{\longleftrightarrow} y$. If $S=\mathbb{Z}^{d}$, we drop it from the notation. We set $0 \longleftrightarrow \infty$ (resp. $0 \longleftrightarrow \partial \Lambda_{n}$ ) if 0 is connected to infinity (resp. 0 is connected to a vertex in $\partial \Lambda_{n}$ ). 
Phase transition. A new idea of this paper is to use a different definition of the critical parameter than the standard one. This new definition relies on the following quantity. For $p \in[0,1]$ and $0 \in S \subset \mathbb{Z}^{d}$, define

$$
\varphi_{p}(S):=p \sum_{\{x, y\} \in \Delta S} \mathbb{P}_{p}[0 \stackrel{S}{\longleftrightarrow} x]
$$

and introduce the following quantities:

$\tilde{p}_{c}:=\sup \left\{p \in[0,1]\right.$ s.t. there exists a finite set $0 \subset S \subset \mathbb{Z}^{d}$ with $\left.\varphi_{p}(S)<1\right\}$,

$p_{c}:=\sup \left\{p\right.$ s.t. $\left.\mathbb{P}_{p}[0 \longleftrightarrow \infty]=0\right\}$.

We are now in a position to state our main result.

Theorem 1.1. For any $d \geq 2, \tilde{p}_{c}=p_{c}$. Furthermore,

1. For $p<p_{c}$, there exists $c=c(p)>0$ such that for every $n \geq 1$,

$$
\mathbb{P}_{p}\left[0 \longleftrightarrow \partial \Lambda_{n}\right] \leq e^{-c n}
$$

2. For $p>p_{c}$,

$$
\mathbb{P}_{p}[0 \longleftrightarrow \infty] \geq \frac{p-p_{c}}{p\left(1-p_{c}\right)}
$$

\section{Remarks.}

1. We refer to [DCT15] for a detailed bibliography, and for a version of the proof valid in greater generality. The aim of this note is to provide a proof in the simplest framework.

2. Theorem 1.1 was proved by Aizenman and Barsky [AB87] in the more general framework of long-range percolation. In their proof, they consider an additional parameter $h$ corresponding to an external field, and they derive the results from differential inequalities satisfied by the thermodynamical quantities of the model. A different proof, based on the geometric study of the pivotal edges, was obtained at the same time by Menshikov [Men86]. These two proofs are also presented in Gri99.

3. In the definition of $\tilde{p}_{c}$, the set of parameters $p$ such that there exists a finite set $0 \subset S \subset \mathbb{Z}^{d}$ with $\varphi_{p}(S)<1$ is an open subset of $[0,1]$. Thus, $\tilde{p}_{c}$ do not belong to this set.

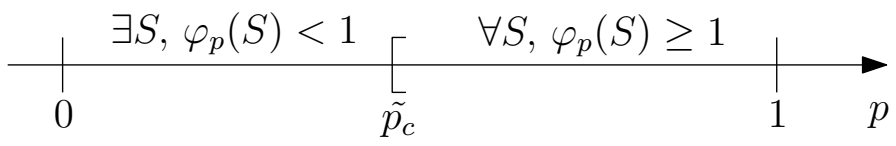


We obtain that the expected size of the cluster of the origin satisfies that for every $p>p_{c}$,

$$
\sum_{x \in \mathbb{Z}^{d}} \mathbb{P}_{p}[0 \longleftrightarrow x] \geq \sum_{n \geq 0} \varphi_{p}\left(\Lambda_{n}\right)=+\infty
$$

4. Since $\varphi_{p}(\{0\})=2 d p$, we obtain $p_{c} \geq 1 / 2 d$.

5. Item 2 provides a mean-field lower bound for the infinite cluster density.

6. Theorem 1.1 implies that $p_{c} \leq 1 / 2$ on $\mathbb{Z}^{2}$. Combined with Zhang's argument [Gri99, Lemma 11.12], this shows that $p_{c}=1 / 2$.

\section{Proof of the theorem}

It is sufficient to show Items 1 and 2 with $p_{c}$ replaced by $\tilde{p}_{c}$ (since it immediately implies the equality $p_{c}=\tilde{p}_{c}$ ).

\subsection{Proof of Item 1}

The proof of Item 1 can be derived from the BK-inequality vdBK85. We present here an exploration argument, similar to the one in [Ham57, which does not rely on the BK-inequality. Let $p<\tilde{p}_{c}$. By definition, one can fix a finite set $S$ containing the origin, such that $\varphi_{p}(S)<1$. Let $L>0$ such that $S \subset \Lambda_{L-1}$.

Let $k \geq 1$ and assume that the event $0 \longleftrightarrow \partial \Lambda_{k L}$ holds. Let

$$
\mathscr{C}=\{z \in S: 0 \stackrel{S}{\longleftrightarrow} z\} .
$$

Since $S \cap \partial \Lambda_{k L}=\varnothing$, there exists an edge $\{x, y\} \in \Delta S$ such that the following events occur:

- 0 is connected to $x$ in $S$,

- $\{x, y\}$ is open,

- $y$ is connected to $\partial \Lambda_{k L}$ in $\mathscr{C}^{c}$.

Using first the union bound, and then a decomposition with respect to possible values of $\mathscr{C}$, we find

$$
\begin{aligned}
& \mathbb{P}_{p}\left[0 \longleftrightarrow \partial \Lambda_{k L}\right] \\
& \leq \sum_{\{x, y\} \in \Delta S} \sum_{C \subset S} \mathbb{P}_{p}\left[\{0 \stackrel{S}{\longleftrightarrow} x, \mathscr{C}=C\} \cap\{\{x, y\} \text { is open }\} \cap\left\{y \stackrel{\mathbb{Z}^{d} \backslash C}{\longleftrightarrow} \partial \Lambda_{k L}\right\}\right] \\
& =p \sum_{\{x, y\} \in \Delta S} \sum_{C \subset S} \mathbb{P}_{p}[0 \stackrel{S}{\longleftrightarrow} x, \mathscr{C}=C] \mathbb{P}_{p}\left[y \stackrel{\mathbb{Z}^{d} \backslash C}{\longleftrightarrow} \partial \Lambda_{k L}\right] .
\end{aligned}
$$


In the second line, we used the fact that the three events depend on different sets of edges and are therefore independent. Since $y \in \Lambda_{L}$, one can bound $\mathbb{P}_{p}\left[y \stackrel{\mathbb{Z}^{d} \backslash C}{\longleftrightarrow} \partial \Lambda_{k L}\right]$ by $\mathbb{P}_{p}\left[0 \longleftrightarrow \partial \Lambda_{(k-1) L}\right]$ in the last expression. Hence, we find

$$
\mathbb{P}_{p}\left[0 \longleftrightarrow \partial \Lambda_{k L}\right] \leq \varphi_{p}(S) \mathbb{P}_{p}\left[y \longleftrightarrow \partial \Lambda_{(k-1) L}\right]
$$

which by induction gives

$$
\mathbb{P}_{p}\left[0 \longleftrightarrow \partial \Lambda_{k L}\right] \leq \varphi_{p}(S)^{k-1}
$$

This proves the desired exponential decay.

\subsection{Proof of Item 2}

Let us start by the following lemma providing a differential inequality valid for every $p$.

Lemma 2.1. Let $p \in[0,1]$ and $n \geq 1$,

$$
\frac{d}{d p} \mathbb{P}_{p}\left[0 \longleftrightarrow \partial \Lambda_{n}\right] \geq \frac{1}{p(1-p)} \cdot \inf _{\substack{S \subset \Lambda_{n} \\ 0 \in S}} \varphi_{p}(S) \cdot\left(1-\mathbb{P}_{p}\left[0 \longleftrightarrow \partial \Lambda_{n}\right]\right)
$$

Let us first see how it implies Item 2 of Theorem 1.1. Integrating the differential inequality (2.1) between $\tilde{p}_{c}$ and $p>\tilde{p}_{c}$ implies that for every $n \geq 1, \mathbb{P}_{p}\left[0 \longleftrightarrow \partial \Lambda_{n}\right] \geq \frac{p-\tilde{p}_{c}}{p\left(1-\tilde{p}_{c}\right)}$. By letting $n$ tend to infinity, we obtain the desired lower bound on $\mathbb{P}_{p}[0 \longleftrightarrow \infty]$.

Proof of Lemma [2.1. Recall that $\{x, y\}$ is pivotal for the configuration $\omega$ and the event $\left\{0 \longleftrightarrow \partial \Lambda_{n}\right\}$ if $\omega_{\{x, y\}} \notin\left\{0 \longleftrightarrow \partial \Lambda_{n}\right\}$ and $\omega\{x, y\} \in\left\{0 \longleftrightarrow \partial \Lambda_{n}\right\}$. (The configuration $\omega_{\{x, y\}}$, resp. $\omega^{\{x, y\}}$, coincides with $\omega$ except that the edge $\{x, y\}$ is closed, resp. open.) By Russo's formula (see Gri99, Section $2.4]$ ), we have

$$
\begin{aligned}
\frac{d}{d p} \mathbb{P}_{p}\left[0 \longleftrightarrow \partial \Lambda_{n}\right] & =\sum_{e \subset \Lambda_{n}} \mathbb{P}_{p}[e \text { is pivotal }] \\
& =\frac{1}{1-p} \sum_{e \subset \Lambda_{n}} \mathbb{P}_{p}\left[e \text { is pivotal, } 0 \leftrightarrow \partial \Lambda_{n}\right]
\end{aligned}
$$

Define the following random subset of $\Lambda_{n}$ :

$$
\mathscr{S}:=\left\{x \in \Lambda_{n} \text { such that } x \leftrightarrow \partial \Lambda_{n}\right\} .
$$

The boundary of $\mathscr{S}$ corresponds to the outmost blocking surface (which can be obtained by exploring from the outside the set of vertices connected to the boundary). When 0 is not connected to $\partial \Lambda_{n}$, the set $\mathscr{S}$ is always 
a subset of $\Lambda_{n}$ containing the origin. By summing over the possible values for $\mathscr{S}$, we obtain

$$
\frac{d}{d p} \mathbb{P}_{p}\left[0 \longleftrightarrow \partial \Lambda_{n}\right]=\frac{1}{1-p} \sum_{\substack{S \subset \Lambda_{n} \\ 0 \in S}} \sum_{e \in \Lambda_{n}} \mathbb{P}_{p}[e \text { is pivotal, } \mathscr{S}=S]
$$

Observe that on the event $\mathscr{S}=S$, the pivotal edges are the edges $\{x, y\} \in$ $\Delta S$ such that 0 is connected to $x$ in $S$. This implies that

$$
\frac{d}{d p} \mathbb{P}_{p}\left[0 \longleftrightarrow \partial \Lambda_{n}\right]=\frac{1}{1-p} \sum_{\substack{S \subset \Lambda_{n} \\ 0 \in S}} \sum_{\{x, y\} \in \Delta S} \mathbb{P}_{p}[0 \stackrel{S}{\longleftrightarrow} x, \mathscr{S}=S]
$$

The event $\{\mathscr{S}=S\}$ is measurable with respect to the configuration outside $S$ and is therefore independent of $\{0 \stackrel{S}{\longleftrightarrow} x\}$. We obtain

$$
\begin{aligned}
\frac{d}{d p} \mathbb{P}_{p}\left[0 \longleftrightarrow \partial \Lambda_{n}\right] & =\frac{1}{1-p} \sum_{\substack{S \subset \Lambda_{n} \\
0 \in S}} \sum_{\{x, y\} \in \Delta S} \mathbb{P}_{p}[0 \stackrel{S}{\longleftrightarrow} x] \mathbb{P}_{p}[\mathscr{S}=S] \\
& =\frac{1}{p(1-p)} \sum_{\substack{S \subset \Lambda_{n} \\
0 \in S}} \varphi_{p}(S) \mathbb{P}_{p}[\mathscr{S}=S] \\
& \geq \frac{1}{p(1-p)} \inf _{\substack{S \subset \Lambda_{n} \\
0 \in S}} \varphi_{p}(S) \cdot \mathbb{P}_{p}\left[0 \leftrightarrow \partial \Lambda_{n}\right],
\end{aligned}
$$

as desired.

Acknowledgments This work was supported by a grant from the Swiss FNS and the NCCR SwissMap also founded by the swiss NSF.

\section{References}

[AB87] Michael Aizenman and David J. Barsky. Sharpness of the phase transition in percolation models. Comm. Math. Phys., 108(3):489-526, 1987.

[DCT15] Hugo Duminil-Copin and Vincent Tassion. A new proof of the sharpness of the phase transition for Bernoulli percolation and the Ising model. preprint, 2015.

[Gri99] Geoffrey Grimmett. Percolation, volume 321 of Grundlehren der Mathematischen Wissenschaften /Fundamental Principles of Mathematical Sciences]. Springer-Verlag, Berlin, second edition, 1999. 
[Ham57] J. M. Hammersley. Percolation processes: Lower bounds for the critical probability. Ann. Math. Statist., 28:790-795, 1957.

[Men86] M. V. Menshikov. Coincidence of critical points in percolation problems. Dokl. Akad. Nauk SSSR, 288(6):1308-1311, 1986.

[vdBK85] J. van den Berg and H. Kesten. Inequalities with applications to percolation and reliability. J. Appl. Probab., 22(3):556-569, 1985.

Département de Mathématiques Université de Genève Genève, SWITZERLAND E-MAIL: hugo.duminil@unige.ch, vincent.tassion@unige.ch 